\title{
Statistical nuclear properties and synthesis of ${ }^{138} \mathrm{La}$
}

\author{
B. V. Kheswa ${ }^{1,2, a}$, M. Wiedeking ${ }^{2}$, F. Giacoppo ${ }^{3}$, S. Goriely ${ }^{4}$, M. Guttormsen ${ }^{3}$, A. C. Larsen ${ }^{3}$, F. L. Bello Garrote $^{3}$, \\ T. K. Eriksen ${ }^{3}$, A. Görgen ${ }^{3}$, T. W. Hagen ${ }^{3}$, P. E. Koehler ${ }^{3}$, M. Klintefjord ${ }^{3}$, H. T. Nyhus ${ }^{3}$, P. Papka ${ }^{3}$, T. Renstrøm $^{3}$, \\ S. Rose ${ }^{3}$, E. Sahin ${ }^{3}$, S. Siem ${ }^{3}$, and T. Tornyi ${ }^{3}$ \\ ${ }^{1}$ iThemba LABS, P.O. Box 722, 7129 Somerset West, South Africa \\ ${ }^{2}$ Physics Department, University of Stellenbosch, Private Bag X1, Matieland 7602, Stellenbosch, South Africa \\ ${ }^{3}$ Department of Physics, University of Oslo, N-0316 Oslo, Norway \\ ${ }^{4}$ Institut d'Astronomie et d'Astrophysique, Université Libre de Bruxelles, CP 226, B-1050 Brussels, Belgium
}

\begin{abstract}
The synthesis of the neutron deficient ${ }^{138} \mathrm{La}$ nucleus has been a puzzle for a long time. It has not been clear whether it is produced through photodisintegration processes or neutrino induced reactions due to unavailability of experimental data for nuclear level densities and $\gamma$ strength functions of ${ }^{138,139}$ La nuclei. In the present work these nuclear properties have been measured and are used to investigate the synthesis of ${ }^{138} \mathrm{La}$. The results support the neutrino interactions as a dominant production process for ${ }^{138} \mathrm{La}$.
\end{abstract}

\section{Introduction}

Most $p$-nuclei are synthesized in the $\mathrm{O} / \mathrm{Ne}$-rich layers of Type II supernovae through photodisintegration ( $p$ process) of $s$ - and $r$-process seed nuclei [1]. ${ }^{138} \mathrm{La}$ is one of these $p$-nuclei, but its solar abundance is under-produced in $p$-process calculations by a factor of $\approx 10$ [1], and more exotic processes such as neutrino-induced reactions have been evoked to explain its synthesis [2,3]. The solar abundance of ${ }^{138} \mathrm{La}$ can be reproduced through charged-current neutrino interactions with ${ }^{138} \mathrm{Ba}$, as quantitatively shown by Ref. [1]. Nevertheless, the significance of the $p$-process cannot be ruled out due to limited knowledge and uncertainties of the nuclear level densities (NLD) and $\gamma$ strength functions $(\gamma \mathrm{SF})$ of the ${ }^{138,139} \mathrm{La}$ nuclei, which are critical ingredients to the Hauser-Feshbach model on which the astrophysical reaction rate calculations are based. It has been pointed out in Ref. [1] that the errors related to these nuclear properties can amount to a factor of $\approx 2$ for the production and destruction of ${ }^{138} \mathrm{La}$ at the relevant $p$-process peak temperature of $2.5 \times 10^{9} \mathrm{~K}$. These statistical nuclear parameters have never been measured below the neutron separation energy for ${ }^{138} \mathrm{La}$ or below $6 \mathrm{MeV}$ for ${ }^{139} \mathrm{La} \mathrm{[4],}$ which is the $\gamma$ ray energy region of importance for the synthesis of ${ }^{138} \mathrm{La}$.

Clearly, additional nuclear physics information is needed to improve our understanding of ${ }^{138} \mathrm{La}$ production in the cosmos. Here we present the experimental $\gamma \mathrm{SF}$ and NLD of ${ }^{138,139} \mathrm{La}$ that have been recently measured using the Oslo Method. Their impact on the galactic synthesis of ${ }^{138} \mathrm{La}$ is also briefly discussed.

\footnotetext{
a e-mail: vincent@tlabs.ac.za
}

\section{Experimental Details}

The experiment was conducted at the Olso Cyclotron Laboratory with a ${ }^{3} \mathrm{He}$ beam of $38 \mathrm{MeV}$ impinging on a 2.5 $\mathrm{mg} / \mathrm{cm}^{2}$ thick natural ${ }^{139} \mathrm{La}(99.9 \%)$ target. The beam intensity was kept in the range $\approx 0.4-0.7 \mathrm{pnA}$. Excited ${ }^{138,139} \mathrm{La}$ nuclei were produced in the ${ }^{139} \mathrm{La}\left({ }^{3} \mathrm{He}, \alpha \gamma\right)$ and ${ }^{139} \mathrm{La}\left({ }^{3} \mathrm{He},{ }^{3} \mathrm{He}^{\prime} \gamma\right)$ reactions, respectively. Particles- $\gamma$ coincidence events were measured using the CACTUS (26 $\mathrm{Nal}(\mathrm{Ti})$ detectors) and SiRi ( $\triangle \mathrm{E}$-E silicon telescope with $130 \mu \mathrm{m}$ thick, 8-channel, $\Delta \mathrm{E}$ and $1550 \mu \mathrm{m}$ thick, single channel, E detectors) arrays [5, 6]. The CACTUS array is mounted such that the target is located at the center with a distance of $22 \mathrm{~cm}$ from each NaI detector. The SiRi array was placed $50 \mathrm{~mm}$ downstream from the target and covers a mean scattering angular range of $40-54^{\circ}$ with a total of 64 channels of which the outermost channels were not included in the analysis.

The $\gamma \mathrm{SF}$ and NLD were extracted simultaneously using the Oslo Method [7, 8]. The excitation energy, $E_{x}$, of the ${ }^{138,139}$ La nuclei were computed from the measured total energy of ${ }^{3} \mathrm{He}$ and $\alpha$ particles, using kinematics and corresponding Q-values. The $E_{x}$ vs $E_{\gamma}$ matrices for ${ }^{138,139} \mathrm{La}$ were extracted from particle $\gamma$-ray coincidence events, which were obtained within an $\approx 50$ ns coincidence window. These matrices were unfolded, with the CACTUS response matrix and iterative technique discussed in [9], to eliminate the contribution from Compton scattering and pair production and only retain the contribution from full-energy depositions. From these, the first generation matrices, $P\left(E_{x}, E_{\gamma}\right)$, were extracted using the first generation method [10]. Figure 1 shows the first generation matrix for ${ }^{139} \mathrm{La}$. The valley with no data in this matrix corresponds to the $1043 \mathrm{keV}$ gap between the first- and 
second-excited states of ${ }^{139} \mathrm{La}$. A similar first generation matrix was generated for ${ }^{138} \mathrm{La}$.

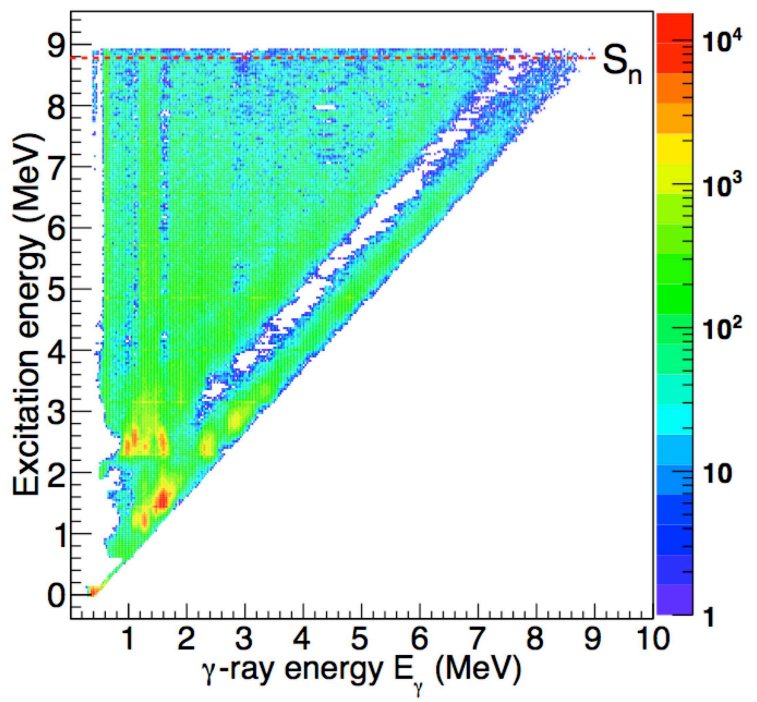

Figure 1. First generation matrix for ${ }^{139} \mathrm{La}$. The horizontal red line indicates the neutron separation energy, $S_{n}$.

We assume that a residual nucleus reaches a compound-like state before emitting a $\gamma$-ray and that the Brink Hypothesis [11] is valid. It follows that in the quasicontinuum region the first generation matrices, $P\left(E_{x}, E_{\gamma}\right)$, can be factorized as [7],

$$
P\left(E_{x}, E_{\gamma}\right) \propto \tilde{\rho}\left(E_{f}\right) \tilde{\mathcal{T}}\left(E_{\gamma}\right)
$$

where $\tilde{\mathcal{T}}\left(E_{\gamma}\right)$ and $\tilde{\rho}\left(E_{f}\right)$ are the $\gamma$-ray transmission coefficient and level density at $E_{f}=E_{x}-E_{\gamma}$, respectively. These statistical parameters are extracted by fitting theoretical first generation matrices $P_{t h}\left(E_{x}, E_{\gamma}\right)$ to the experimental $P\left(E_{x}, E_{\gamma}\right)$ according to the iterative procedure presented in Ref. [7]. This was performed in the energy regions of $E_{\gamma} \geq 1 \mathrm{MeV}$ and $3.5 \mathrm{MeV} \leq E_{x} \leq 7.1 \mathrm{MeV}$ for ${ }^{138} \mathrm{La}$, and $E_{\gamma} \geq 1.7 \mathrm{MeV}$ and $3.5 \mathrm{MeV} \leq E_{x} \leq 8.5 \mathrm{MeV}$ for ${ }^{139} \mathrm{La}$, to exclude the non-statistical excitation energy regions.

Once extracted, infinitely many solutions of $P\left(E_{x}, E_{\gamma}\right)$ can be obtained of the form

$$
\begin{gathered}
\rho\left(E_{f}\right)=A \tilde{\rho}\left(E_{f}\right) e^{\alpha E_{f}} \\
\mathcal{T}\left(E_{\gamma}\right)=B \tilde{\mathcal{T}}\left(E_{\gamma}\right) e^{\alpha E_{\gamma}} .
\end{gathered}
$$

The $\alpha$ parameter is the common slope for $\rho\left(E_{f}\right)$ and $\mathcal{T}\left(E_{\gamma}\right)$ and $A$ and $B$ are normalization parameters. These parameters were obtained following the procedure presented in details in Ref. [12].

Assuming that statistical decay is dominated by dipole transitions the $\gamma \mathrm{SF}, f\left(E_{\gamma}\right)$, can be calculated from $\mathcal{T}\left(E_{\gamma}\right)$ according to

$$
f\left(E_{\gamma}\right)=\frac{\mathcal{T}\left(E_{\gamma}\right)}{2 \pi E_{\gamma}^{3}} .
$$

\section{Results and Discussion}

The $f\left(E_{\gamma}\right)$ and $\rho\left(E_{f}\right)$ extracted for both La nuclei are shown in figures 2 and 3. The error bars in these figures reflect statistical errors and systematic uncertainties from the unfolding of the NaI detectors and from the first generation matrices, see Sec. 2.2.4 in Ref. [7]. The $f\left(E_{\gamma}\right)$ show interesting and unexpected features. In particular the ${ }^{138} \mathrm{La}$ $f\left(E_{\gamma}\right)$ exhibits a pronounced up-bend at $E_{\gamma}<1.7 \mathrm{MeV}$ and a plateau behavior in the range $1.7 \mathrm{MeV}<E_{\gamma}<4$ $\mathrm{MeV}$. This up-bend is being observed for the first time in the mass region $A>106$ [13-18]. The physical origin of the up-bend is still debatable, but two different theoretical predictions have been recently reported. According to Ref. [19] the enhancement is due to M1 transitions from the reorientation of the spins of high-j neutron and proton orbits, while Ref. [20] suggests that E1 radiation resulting from transitions from the quasicontinuum to the singleparticle continuum may be responsible for the enhanced strength. However, it is interesting to note that ${ }^{138} \mathrm{La}$ is located within a predicted region of magnetic rotation [21], which is consistent with Ref. [19]. Furthermore, the ${ }^{139} \mathrm{La}$ $f\left(E_{\gamma}\right)$ shows resonance behaviour at $E_{\gamma} \approx 6.4 \mathrm{MeV}$ which was reported prior to this work and discussed to be an E1 pygmy resonance [4].

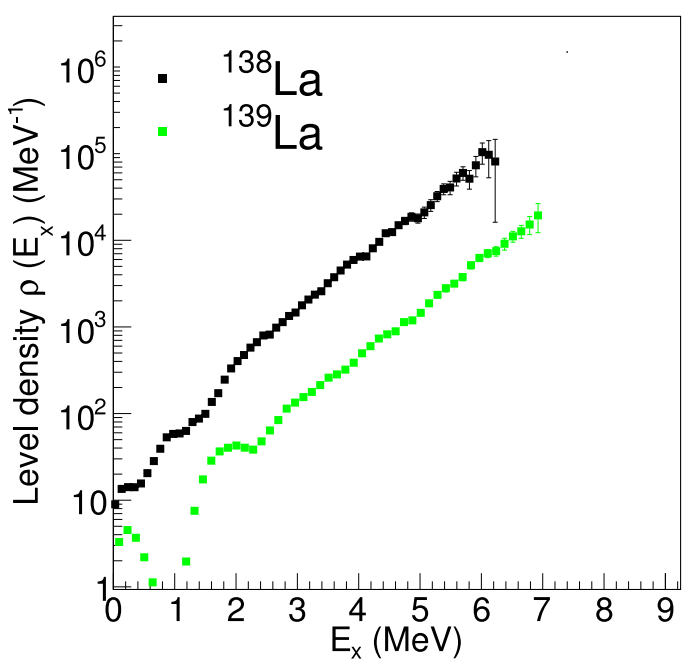

Figure 2. The nuclear level densities of ${ }^{138,139} \mathrm{La}$.

To investigate the $p$-process production of ${ }^{138} \mathrm{La}$, the experimental $f\left(E_{\gamma}\right)$ and $\rho$, calculated with the HartreeFock-Bogoliubov (HFB) plus combinatorial model, were used to calculate the corresponding Maxwellian averaged cross sections (MACS), at the $p$-process peak temperature $T=2.5 \times 10^{9} \mathrm{~K}$. The $\rho$ obtained from the HFB plus combinatorial model is in good agreement with our experimental values. The calculated MACS are given in table 1 for ${ }^{138} \mathrm{La}(n, \gamma)$ and ${ }^{137} \mathrm{La}(n, \gamma)$ reactions.

At $T=2.5 \times 10^{9} \mathrm{~K}$, the MACS are within a few tens of a percent to the values derived previously in Ref. [1] (see table 1). In relation to the ${ }^{138} \mathrm{La}$ synthesis study performed in Ref. [1], on the basis of detailed balance rela- 


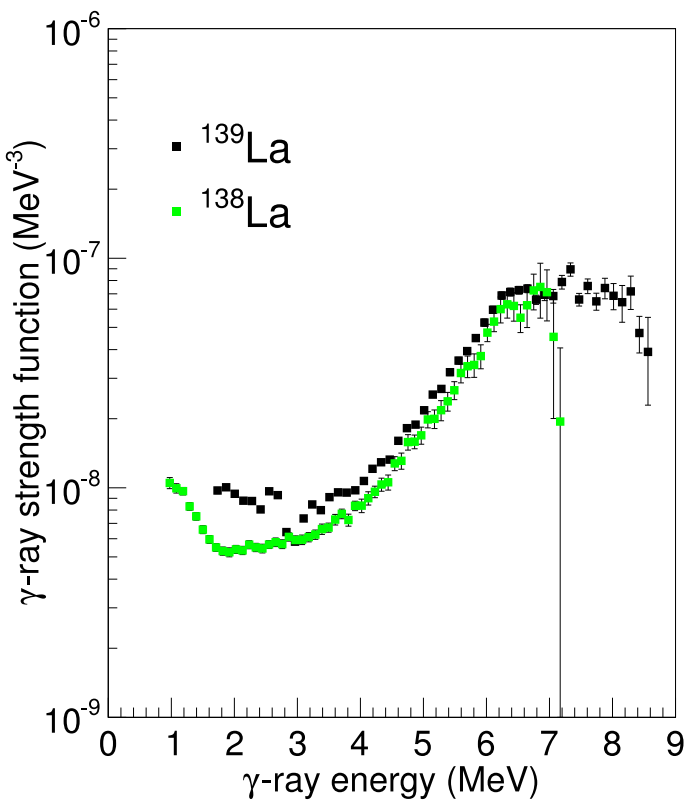

Figure 3. The $\gamma$ ray strength functions of ${ }^{138,139} \mathrm{La}$.

tions, the ${ }^{139} \mathrm{La}(\gamma, \mathrm{n}){ }^{138} \mathrm{La}$ production rate is smaller and the ${ }^{138} \mathrm{La}(\gamma, \mathrm{n}){ }^{137} \mathrm{La}$ destruction larger than predicted in that study. The new determination of the reaction rates with the improved $\gamma \mathrm{SFs}$ therefore shows that ${ }^{138} \mathrm{La}$ cannot be produced by photoreactions during the standard $p$ process and strongly supports the neutrino process as the dominant production process for ${ }^{138} \mathrm{La}$.

Table 1. Maxwellian averaged cross sections calculated from experimental results and taken from Ref. [1].

\begin{tabular}{llll}
\hline Reaction & $\mathrm{T}(\mathrm{K})$ & MACS $(\mathrm{mb})$ & MACS $(\mathrm{mb})$ \\
\hline${ }^{138} \mathrm{La}(n, \gamma)$ & $2.5 \times 10^{9}$ & $54 \pm 20$ & $62[1]$ \\
${ }^{137} \mathrm{La}(n, \gamma)$ & $2.5 \times 10^{9}$ & $116 \pm 44$ & $123[1]$ \\
\hline
\end{tabular}

\section{Conclusions}

We have measured the $\gamma$ strength functions and nuclear level densities for ${ }^{138,139} \mathrm{La}$. From these results the MACS were calculated and from these, the ${ }^{139} \mathrm{La}(\gamma, n)^{138} \mathrm{La}$ production rate was found to be smaller and the ${ }^{138} \mathrm{La}(\gamma, n){ }^{137} \mathrm{La}$ destruction rate larger. The new determination of the reaction rates therefore shows that ${ }^{138} \mathrm{La}$ cannot be produced by photoreactions during the standard $p$-process. This result puts the neutrino process as the dominant production process for ${ }^{138} \mathrm{La}$ on a very strong footing.

\section{Acknowledgements}

We thank J. C. Müller, A. Semchenkov, and J. C. Wikne for providing excellent beam quality throughout the experiment and N.Y. Kheswa for manufacturing the target. This work is based upon support by the National Research Foundation of South Africa. This work was partially funded by the Research Council of Norway, project grant no. 205528, 213442, and 210007. S.G. grants the support of the F.R.S.-FNRS.

\section{References}

[1] S. Goriely et al., A\&A 375, L35 (2001).

[2] S.E. Woosley et al., Astrophys. J. 356, 272 (1990).

[3] T. Kajino et al., J. Phys. G: Nucl. Part. Phys. 41, 044007 (2014).

[4] A. Makinaga et al., Phys. Rev. C. 82, 024314 (2010).

[5] M. Guttormsen et al., Nucl. Instrum. Methods Phys. Res. A 648, (2011).

[6] M. Guttormsen et al., Phys. Scr. T32, 54 (1990).

[7] A. Schiller et al., Nucl. Instrum. Methods Phys. Res. A 447, 498 (2000).

[8] A.C. Larsen et al., Phys. Rev. C 83, 034315 (2011).

[9] M. Guttormsen et al., Nucl. Instrum. Methods Phys. Res. A 374, 371 (1996).

[10] M. Guttormsen et al., Nucl. Instrum. Methods Phys. Res. A 255, (1987).

[11] D. M. Brink, Ph.D. thesis, Oxford University, 1955.

[12] B. V. Kheswa, Ph.D. thesis, Stellenbosch University, 2014.

[13] A.C. Larsen et al., Phys. Rev. C 87, 014319 (2013).

[14] A.C. Larsen et al., Phys. Rev. C 73, 064301 (2006).

[15] M. Guttormsen et al., Phys. Rev. Lett. 109, 162503 (2012).

[16] H. K. Toft et al., Phys. Rev. C. 81, 064311 (2010).

[17] A. Voinov et al., Phys. Rev. Lett. 93, 14 (2004).

[18] M. Guttormsen et al., Phys. Rev. C. 71, 044307 (2005).

[19] R. Schwengner, S. Frauendorf, and A.C. Larsen, Phys. Rev. Lett. 111, 232504 (2013).

[20] E. Litvinova and N. Belov, Phys. Rev. C 88, 031302(R) (2013).

[21] S. Frauendorf, Rev. Mod. Phys 73, 463 (2001). 
